Faculty of Engineering

Faculty Publications

An Improved Algorithm for Discrete L1 Linear Approximation

Ian Barrodale and F.D.K. Roberts

MRC Technical Summary Report \#1172

January 1972

A revised version of this internal report was later published in Society for Industrial and Applied Mathematics (SIAM) Journal on Numerical Analysis:

October 1973, 10(5), 839-848.

https://epubs.siam.org/doi/abs/10.1137/0710069

and its Fortran Subroutine was later published in Communications of the ACM, as Algorithm 478:

June $1974,17(6), 319-320$.

https://doi.org/10.1145/355616.361024

Citation for this paper:

Barrodale, I. \& Roberts, F.D.K. (1972). An Improved Algorithm for Discrete L1 Linear Approximation. MRC Technical Summary Report \#1172. 


\section{THE UNIVERSITY OF WISCONSIN}

MATHEMATICS RESEARCH CENTER

Contract No.: DA-31-124-ARO-D-462

AN IMPROVED ALGORITHM FOR DISCRETE

$$
\ell_{1} \text { IINEAR APPROXIMATION }
$$

I. Barrodale and F. D. K. Roberts

This document has been approved for public release and sale; its distribution is unlimited.

MRC Technical Summary Report \# 1172

January 1972

Received August 17, 1971 


\section{ABSTRACT}

By modifying the simplex method of linear programming, we are able to present an algorithm for $\ell_{1}$ approximation which appears to be superior computationally to any other known algorithm for this problem. 
AN IMPROVED ALGORITHM FOR DISCRETE $\ell_{1}$ LINEAR APPROXIMATION

I. Barrodale ${ }^{*}$ and F. D. K. Roberts ${ }^{*}$

\section{Introduction}

This paper provides an algorithm which appears to be the most efficient yet devised for solving the general $\ell_{1}$ linear approximation problem. The algorithm is a modification of the simplex method applied to the primal formulation of the $\ell_{1}$ problem as a linear program. A Fortran IV program for the algorithm is supplied, and some numerical results are given comparing the computational behavior of this and other $\ell_{1}$ algorithms.

The general $\ell_{1}$ linear approximation problem can be stated as follows. Let $f(x)$ be a given real-valued function defined on a discrete subset $X=\left\{x_{1}, x_{2}, \ldots, x_{m}\right\}$ of Euclidean space $E^{N}$. Given $n(\leq m)$ real-valued functions $\phi_{j}(x)$ defined on $X$, we form a linear approximating function $L(A, x)=\sum_{j=1}^{n} a_{j} \phi_{j}(x)$ for any set $A=\left\{a_{1}, a_{2}, \ldots, a_{n}\right\}$ of real numbers. The $\ell_{1}$ problem is to determine a best approximation $L(A, x)$ which minimizes

$$
\sum_{i=1}^{m}\left|f\left(x_{i}\right)-L\left(A, x_{i}\right)\right|
$$

It is well known that at least one best approximation always exists, and there are now several algorithms for calculating a best approximation. However,

\footnotetext{
Presently visiting the Mathematics Research Center, Madison, Wisconsin, U.S.A. Work sponsored by the United States Army under Contract No.: DA-31-124-ARO-D-462. Nepartment of Mathematics, University of Victoria, Victoria, B. C., Canada.
} 
some of these algorithms require that the set of given functions $\left\{\phi_{1}(x)\right.$, $\left.\phi_{2}(x), \ldots, \phi_{n}(x)\right\}$ be linearly independent on $x$, or that it satisfy the Haar condition on $\mathrm{X}$. This latter condition, which is equivalent to requiring that the given functions be linearly independent on every subset of $n$ distinct elements from $\mathrm{X}$, can be quite restrictive in practice.

As yet, it seems that the only use made of "least-first-power" approximations has been in the analys is of experimental data. Even in this situation, many scientists are unwilling to use estimates of parameters computed in a norm for which few statistical tests are available. However, there is no doubt (see Barrodale [2]) that best $\ell_{1}$ approximations are often superior to best $\ell_{2}$ approximations when estimating the true form of data that contain some very inaccurate observations.

The approximating functions used by experimental scientists for interpreting data are almost never polynomials, and they rarely constitute Haar sets (indeed, continuous functions of more than one independent variable never form a Haar set). If the approximation problem is presented as the equivalent problem of solving an overdetermined system of linear equations, it may be quite difficult to decide on the rank of the given matrix. Consequently, we feel that a datafitting algorithm should impose as few restrictions as possible on the user's choice of approximating function. Since our algorithm is based on the simplex method it can be used with any linear approximating function. 
The connection between linear programming and $\ell_{1}$ approximation was pointed out by Wagner [15] in 1959, although other more direct (but cumbersome) algorithms for the $\ell_{1}$ problem had been proposed much earlier (see Barrodale [2] for references to these).

In 1966 Barrodale and Young [6] described a primal algorithm which takes advantage of the special structure of the linear programming formulation of the $\ell_{1}$ problem. The primal algorithm of the present paper is an improved version of this earlier simplex algorithm; in short, we have been able to reduce significantly the total number of iterations required, by discovering how to pass through several neighboring simplex vertices in a single iteration.

In 1967 Usow [13] presented a descent method which attempts to calculate a best $\ell_{1}$ approximation by locating the lowest vertex of a convex polytope representing the set of all possible $\ell_{1}$ approximations. This iterative algorithm requires that the approximating function be formed from a Haar set, and it also stops prematurely if a certain degenerate situation arises. Usow [14] states that he has now corrected this latter deficiency and extended his algorithm to approximating functions formed from linearly independent sets of given functions. In 1969 Robers and Ben-Israel [11] applied a new method for linear programming to the dual formulation of the $\ell_{1}$ problem. Their new method (which they call interval linear programming) is capable of solving any bounded-variable linear programming problem, and so it is natural to apply it to the $\ell_{1}$ problem in particular. Robers and Robers [12] have now supplied a special version of the general method of [11] which is designed specifically for the $\ell_{1}$ problem. 
Finally, early in 1971 Abdelmalek [1] described an algorithm which determines best $\ell_{1}$ approximations as the limit of best $\ell_{p}$ approximations as $p \rightarrow 1^{+}$. His technique thus obtains a solution to a linear problem by solving a sequence of nonlinear problems.

Clearly, a comparison of the computational behavior of these various algorithms is both desirable and overdue. Consequently, we have conducted some numerical tests on (i) the latest descent method of Usow [14], (ii) the special adaptation by Robers and Robers [12] of the more general method of Robers and Ben-Israel [11], (iii) the bounded-variable simplex method of Dantzig [7] applied to the dual formulation of the $\ell_{1}$ problem, and (iv) our modified standard form of the simplex method applied to the $\ell_{1}$ problem in its primal form. (The numerical results given in Abdelmalek [1] demonstrate that his algorithm cannot possibly be as efficient as those selected above). These numerical tests confirm that our algorithm is the most efficient in general.

The $\ell_{1}$ problem (1) is restated as a linear program in $\S 2$, our algorithm is described in $\S 3$, and the numerical results are in $\S 4$.

\section{Linear programming and $\ell_{1}$ approximation}

For the $\ell_{1}$ problem (l) let us write $\phi_{j, i} \equiv \phi_{j}\left(x_{i}\right), f_{i} \equiv f\left(x_{i}\right)$, and define nonnegative variables $u_{i}, v_{i}, b_{j}, c_{j}$ by putting

$$
f_{i}-\sum_{j=1}^{n} a_{j} \phi_{j, i}=u_{i}-v_{i}, \quad \text { for } i=1,2, \ldots, m \text {, }
$$

and $a_{j}=b_{j}-c_{j}$ for $j=1,2, \ldots, n$. Then a best $\ell_{1}$ approximation corresponds to an optimal solution to the (primal) linear programming problem: 


$$
\begin{aligned}
& \operatorname{minimize} \sum_{i=1}^{m}\left(u_{i}+v_{i}\right) \\
& \text { subject to } f_{i}=\sum_{j=1}^{n}\left(b_{j}-c_{j}\right) \phi_{j, i}+u_{i}-v_{i} \\
& \qquad(i=1,2, \ldots, m) \\
& \text { and } b_{j}, c_{j}, u_{i}, v_{i} \geq 0 .
\end{aligned}
$$

The formulation (2) appears in Barrodale and Roberts [4] along with the following result.

Theorem. If the column rank of the $m \times n$ matrix $\Phi=\left\{\phi_{j, i}\right\}^{\mathrm{T}}$ is $k(\leq n)$, then there exists a best $\ell_{1}$ approximation which interpolates $f(x)$ in at least $k$ points of $X$.

Several authors (Wagner [15], Rabinowitz [10], Barrodale [3], for example) have suggested that the dual of (2) should be solved instead when $m$ is large. The dual of (2) is stated most conveniently (e.g. Rabinowitz [10]) as the following bounded-variable linear programming problem:

$$
\begin{aligned}
& \operatorname{maximize} \sum_{i=1}^{m}\left(w_{i} f_{i}-f_{i}\right) \\
& \text { subject to } \sum_{i=1}^{m} w_{i} \phi_{j, i}=\sum_{i=1}^{m} \phi_{j, i} \\
& \qquad(j=1,2, \ldots, n) \\
& \text { and } 0 \leq w_{i} \leq 2 .
\end{aligned}
$$


As the results of our numerical tests indicate, applying the bounded-variable simplex method of Dantzig [7] to (3) leads to a less efficient algorithm in general than solving (2) by our version of the standard form of the simplex method.

\section{The algorithm}

A direct application of the simplex method to (2) does not yield an efficient algorithm. (On the other hand, this might well be a reasonable strategy if a particular problem arises involving additional (inequality) constraints on the approximating function). Barrodale and Young [6] observed that an initial basic feasible solution to (2) is immediately available, and that most of the column vectors in the simplex tableau need not be stored explicitly. Barrodale and Roberts [4] recommended that for the first $n$ iterations the choice of pivotal column be restricted to the columns associated with the variables $b_{j}$ and $c_{j}$, and that thereafter a special pivotal column selection rule be employed. In spite of these improvements, the simplex method can take a large number of iterations to solve some problems. The reason for this can best be explained by considering the following particular problem.

Suppose that we wish to approximate $f(x)=e^{x}$ on 201 uniformly spaced points in the interval $[0,2]$ by a straight line $L(A, x)=a_{1}+a_{2} x$. The initial simplex basis is provided by the column vectors associated with

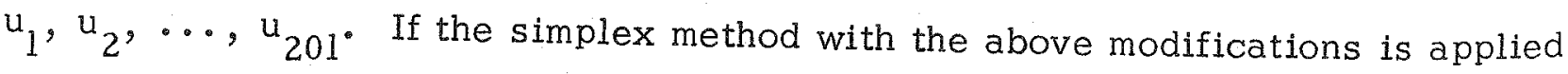
to this problem, the first iteration brings the vector associated with $b_{1}$ into the 
basis and forces the vector associated with $u_{1}$ out. The second iteration brings the vector corresponding to $\mathrm{b}_{2}$ into the basis, and that corresponding to $\mathrm{u}_{2}$ goes out. Thus, after two iterations, the simplex tableau represents an approximation which interpolates the first and second data points (since the current values of $u_{1}, v_{1}, u_{2}$, and $v_{2}$, are all zero). Subsequent iterations correspond to approximations which interpolate the following pairs of data points: (1st., 3rd.), (2nd., 3rd.), (2nd., 4th.), (2nd., 5th.), (2nd.,6th.), (3rd., 6th.), (3rd., 7 th.), .... After 201 iterations, the (unique) best approximation is obtained which interpolates the 51st. and 15lst. data points. The difficulty arises because of the nonnegativity restrictions on $u_{i}, v_{i}$ (and also $b_{j}, c_{j}$ ) which are somewhat artificial. What is required is an algorithm which at each iteration can bypass intermediate data points and thereby substantially reduce the number of iterations required to obtain a best approximation. We shall now describe such an algorithm; it requires just 7 iterations to solve the above problem.

Inspection of the linear programming problem (2) reveals that (i) an initial basic feasible solution is immediately available, and (ii) only $n$ columns are needed to store the information contained in the right-hand sides of the equality constraints. Thus, denoting the columns of the simplex tableau corresponding to (2) by $\underline{R}, \underline{b}_{j}, \underline{c}_{j}, \underline{u}_{i}, \underline{v}_{i}$ (see Table 2 , for example), an initial basis is provided by $\underline{u}_{1}, \underline{u}_{2}, \ldots, \underline{u}_{m}$ whenever each $f_{i}$ is nonnegative. If an $f_{i}$ is negative we change the sign of the corresponding row and replace $\underline{u}_{i}$ in the basis by $\underline{v}_{i}$. It is also clear that 
$\underline{b}_{j}=-\underline{c}_{j}$ and $\underline{u}_{i}=-\underline{v}_{i}$, and that the sum of the marginal (or reduced) costs of $\underline{b}_{j}$ and $\underline{c}_{j}$ is zero and of $\underline{u}_{i}$ and $\underline{v}_{i}$ is -2 . Thus the condensed form of the simplex method (in which the basis is suppressed) can be applied to just $n$ columns, which initially contain $\underline{b}_{1}, \underline{b}_{2}, \ldots, \underline{b}_{n}$.

\begin{tabular}{|c|c|c|c|c|c|}
\hline Basis & $\underline{\mathrm{R}}$ & $\underline{b}_{1}$ & $b_{2}$ & $\cdots$ & $\underline{b}_{n}$ \\
\hline$\underline{u}_{1}$ & $f_{1}$ & $\phi_{1,1}$ & $\phi_{2,1}$ & $\cdots$ & $\phi_{n, 1}$ \\
\hline$\underline{u}_{2}$ & $\mathrm{f}_{2}$ & $\phi_{1,2}$ & $\phi_{2,2}$ & $\cdots$ & $\phi_{n, 2}$ \\
\hline : & : & & & & $\vdots$ \\
\hline$\underline{u}_{m}$ & $\mathrm{f}_{\mathrm{m}}$ & $\phi_{1, \mathrm{~m}}$ & $\phi_{2, m}$ & $\cdots$ & $\phi_{n, m}$ \\
\hline $\begin{array}{l}\text { Marginal } \\
\text { costs }\end{array}$ & $\sum_{i=1}^{m} f_{i}$ & $\sum_{i=1}^{m} \phi_{1, i}$ & $\sum_{i=1}^{m} \phi_{2, i}$ & $\cdots$ & $\sum_{i=1}^{m} \phi_{n, i}$ \\
\hline
\end{tabular}

TABLE 1: Initial condensed simplex tableau for the algorithm, assuming each $f_{i}$ is nonnegative. (A $\underline{\text { full }}$ tableau is displayed in Table 2).

The entire computation is performed using an $m+3$ by $n+2$ matrix. The additional column and one additional row are required to label the vectors (see Table 1), and the remaining row is used whenever the initial tableau is scaled. We scale the initial data by dividing the column vectors $\underline{R}_{,} \underline{b}_{1}$, $\underline{b}_{2}, \ldots, \underline{b}_{n}$ by their largest elements in absolute value. The use of this scaling option in our program is recommended when large problems are to be solved, although it will not always improve their condition. 
The algorithm is implemented in two stages. Stage 1 restricts the choice of pivotal column during the first $n$ iterations to the vectors $\underline{b}_{j}$ and $\underline{c}_{j}$. The vector to enter the basis is chosen as that with the most positive marginal cost. The vector leaving the basis is chosen from among the basic vectors $\underline{u}_{j}$ and $\underline{v}_{i}$ by selecting that which causes the maximum reduction in the objective function. At the end of Stage 1 , the rank $k(\leq n)$ of the matrix $\Phi$ is determined by the total number of vectors $\underline{b}_{j}, \underline{c}_{j}$ in the basis. Since $k$ of the vectors $\underline{u}_{\mathrm{i}}$ (or $\underline{\mathrm{v}}_{\mathrm{i}}$ ) have been removed from the basis, the current simplex tableau represents an approximation which interpolates at least $k$ data points. If the approximation interpolates more than $k$ data points then the simplex tableau is degenerate; this does not cause any problems in practice.

Stage 2 involves interchanging nonbasic $\underline{u}_{i}$ or $\underline{v}_{i}$ with basic $\underline{u}_{i}$ or $\underline{v}_{i}$ : the basic $\underline{b}_{j}$ and $\underline{c}_{j}$ vectors are not allowed to leave the basis during Stage 2 . The vector entering the basis is that with the most positive marginal cost, and the vector leaving the basis is again chosen as that which causes the maximum reduction in the objective function. The algorithm terminates when all the marginal costs are nonpositive. In Stage 2 each simplex tableau corresponds to an approximation which interpolates $k$ data points (assuming nondegeneracy). At each iteration, $k-1$ of these points remain fixed. The vector entering the basis determines which point is to be dropped from the interpolating set while the vector leaving the basis determines the new point of interpolation. 
The final tableau at the end of Stage 2 may be infeasible since some of the basic vectors $\underline{b}_{j}$ and $\underline{c}_{j}$ can have negative values associated with them. The solution becomes feasible (and hence optimal) by interchanging such basic vectors $\underline{b}_{j}$ (or $\underline{c}_{j}$ ) with the corresponding nonbasic vectors $\underline{c}_{j}$ (or $\underline{b}_{j}$ ).

The main modification to the simplex method is in choosing the vector $\underline{u}_{i}$ or $\underline{v}_{i}$ to leave the basis. In both Stage 1 and Stage 2 this vector is chosen as that which causes the maximum reduction in the objective function. A direct search over all the basic vectors $\underline{u}_{i}$ and $\underline{v}_{i}$ is inefficient and a computationally more efficient technique can be used. This is best described by means of a simple worked example.

Example. Find the best $\ell_{1}$ approximation to $\{(1,1),(2,1),(3,2),(4,3)$, $(5,2)\}$ by $L(A, x)=a_{1}+a_{2} x$. Equivalently, find the $\ell_{1}$ solution to the overdetermined system of equations:

$$
\begin{aligned}
& a_{1}+a_{2}=1 \\
& a_{1}+2 a_{2}=1 \\
& a_{1}+3 a_{2}=2 \\
& a_{1}+4 a_{2}=3 \\
& a_{1}+5 a_{2}=2
\end{aligned}
$$

The full initial simplex tableau (without scaling) is given in Table 2. 


\begin{tabular}{|c|c|c|c|c|c|c|c|c|c|c|c|c|c|c|c|c|}
\hline Costs & $\rightarrow$ & & 0 & 0 & & 0 & 1 & 1 & 1 & 1 & 1 & 1 & 1 & 1 & 1 & 1 \\
\hline$\downarrow$ & Basis & $\underline{\mathrm{R}}$ & $\underline{b}_{1}$ & $\underline{b}_{2}$ & $\mathrm{c}_{1}$ & $\mathrm{c}_{2}$ & $\underline{u}_{1}$ & $\underline{u}_{2}$ & $\underline{u}_{3}$ & $\underline{u}_{4}$ & $\underline{u}_{5}$ & $\underline{v}_{1}$ & $\underline{v}_{2}$ & $\underline{v}_{3}$ & $\underline{v}_{4}$ & $\underline{v}_{5}$ \\
\hline 1 & $\underline{u}_{1}$ & 1 & 1 & 1 & -1 & -1 & 1 & & & & & -1 & & & & \\
\hline 1 & $\underline{\mathrm{u}}_{2}$ & 1 & 1 & $2^{* * *}$ & -1 & -2 & & 1 & & & & & -1 & & & \\
\hline 1 & $\underline{\mathrm{u}}_{3}$ & 2 & 1 & $3^{* * * * *}$ & -1 & -3 & & & 1 & & & & & -1 & & \\
\hline 1 & $\underline{u}_{4}$ & 3 & 1 & 4 & -1 & -4 & & & & 1 & & & & & -1 & \\
\hline 1 & $\underline{\mathrm{u}}_{5}$ & 2 & 1 & $5^{*}$ & -1 & -5 & & & & & 1 & & & & & -1 \\
\hline \multicolumn{2}{|c|}{$\begin{array}{r}\text { Marginal } \\
\text { Costs }\end{array} \rightarrow$} & 9 & 5 & 15 & -5 & -15 & 0 & 0 & 0 & 0 & 0 & -2 & -2 & -2 & -2 & -2 \\
\hline
\end{tabular}

TABLE 2: Full initial simplex tableau for the worked example.

At the first iteration in Stage $1, \underline{b}_{2}$ is brought into the basis corresponding to the largest marginal cost $(=15)$. The normal simplex pivot $\left(5^{*}\right)$ corresponds to an approximation in which $b_{2}=2 / 5, u_{5}=v_{5}=0$, i.e. an approximation which interpolates the fifth data point. However, if we increase $b_{2}$ beyond the value $2 / 5$ we can further reduce the objective function, but this makes $u_{5}$ negative. Hence we replace $\underline{u}_{5}$ in the basis by $\underline{v}_{5}$. This can be accomplished by subtracting twice the fifth row from the marginal cost row (thus making the marginal cost of $\underline{v}_{5}$ zero), changing the sign of the fifth row and replacing the label $\underline{\mathrm{u}}_{5}$ by $\underline{\mathrm{v}}_{5}$ in the basis. The marginal cost of $\underline{\mathrm{b}}_{2}$ is now 5 , and hence we increase $\mathrm{b}_{2}$ further. The second pivot $\left(2^{* *_{*}^{*}}\right)$ corresponds to an approximation in which $b_{2}=1 / 2, u_{2}=v_{2}=0, i . e$ an approximation which interpolates the second data point. Interchanging $\underline{u}_{2}$ and $\underline{v}_{2}$ in the basis reduces the marginal 
cost of $\underline{b}_{2}$ to 1. Thus we increase $b_{2}$ further. The third pivot $\left(3^{* * * x \cdot x^{*}}\right)$ corresponds to an approximation in which $b_{2}=2 / 3, u_{3}=v_{3}=0$, i.e. an approximation which interpolates the third data point. The objective function cannot be decreased further by increasing $\mathrm{b}_{2}$, since if we interchange $\underline{u}_{3}$ and $\underline{v}_{3}$ in the basis, the marginal cost of $\underline{b}_{2}$ becomes -5 . Hence we pivot on this element $\left(3^{* * * * *}\right)$ and bring $\underline{b}_{2}$ into the basis in place of $\underline{u}_{3}$. Using the condensed tableau, the complete solution to this example is given in Table 3. After two iterations (at the end of Stage 1) the marginal costs of $\underline{u}_{1}$ and $\underline{u}_{3}$ are -1 and 0 respectively, and so the marginal costs of the (suppressed) vectors $\underline{v}_{1}$ and $\underline{v}_{3}$ are -1 and -2 respectively (recall that the sum of the marginal costs of $\underline{u}_{i}$ and $\underline{v}_{i}$ is -2 ). Since all of the nonbasic vectors have nonpositive marginal costs, there is no need for Stage 2 in this example. The final tableau represents a best approximation $L\left(A^{*}, x\right)=\frac{1}{2}+\frac{1}{2} x$ which interpolates the first and third data points. The deviations at the other points are given by $v_{2}=\frac{1}{2}, u_{4}=\frac{1}{2}, v_{5}=1$

\begin{tabular}{|c|c|cc|}
\hline Basis & $\underline{R}$ & $\underline{b}_{1}$ & $\underline{b}_{2}$ \\
\hline$\underline{u}_{1}$ & 1 & 1 & 1 \\
$\underline{u}_{2}$ & 1 & 1 & $2^{* * *}$ \\
$\underline{u}_{3}$ & 2 & 1 & $3^{* * * *}$ \\
$\underline{u}_{4}$ & 3 & 1 & 4 \\
$\underline{u}_{5}$ & 2 & 1 & $5^{*}$ \\
\hline $\begin{array}{c}\text { Marginal } \\
\text { costs }\end{array}$ & 9 & 5 & 15 \\
\hline
\end{tabular}

\begin{tabular}{|c|c|cc|}
\hline Basis & $\underline{\mathrm{R}}$ & $\underline{\mathrm{b}}_{1}$ & $\underline{\mathrm{u}}_{3}$ \\
\hline$\underline{\underline{u}}_{\mathrm{l}}$ & $1 / 3$ & $2 / 3$ & $-1 / 3$ \\
$\underline{\mathrm{v}}_{2}$ & $1 / 3$ & $-1 / 3$ & $2 / 3$ \\
$\underline{\mathrm{b}}_{2}$ & $2 / 3$ & $1 / 3$ & $1 / 3$ \\
$\underline{\mathrm{u}}_{4}$ & $1 / 3$ & $-1 / 3$ & $-4 / 3$ \\
$\underline{\mathrm{v}}_{5}$ & $4 / 3$ & $2 / 3$ & $5 / 3$ \\
\hline $\begin{array}{c}\text { Marginal } \\
\text { costs }\end{array}$ & $7 / 3$ & $2 / 3$ & $-1 / 3$ \\
\hline
\end{tabular}

\begin{tabular}{|c|c|cc|}
\hline Basis & $\underline{\mathrm{R}}$ & $\underline{\underline{u}}_{1}$ & $\underline{\mathrm{u}}_{3}$ \\
\hline$\underline{\mathrm{b}}_{1}$ & $1 / 2$ & $3 / 2$ & $-1 / 2$ \\
$\underline{\mathrm{v}}_{2}$ & $1 / 2$ & $1 / 2$ & $1 / 2$ \\
$\underline{\mathrm{b}}_{2}$ & $1 / 2$ & $-1 / 2$ & $1 / 2$ \\
$\underline{\mathrm{u}}_{4}$ & $1 / 2$ & $1 / 2$ & $-3 / 2$ \\
$\underline{\mathrm{v}}_{5}$ & 1 & -1 & 2 \\
\hline $\begin{array}{c}\text { Marginal } \\
\text { costs }\end{array}$ & 2 & -1 & 0 \\
\hline
\end{tabular}

TABLE 3: Solution to the worked example using condensed tableaux. 
and the error of approximation is 2 . The solution is not unique since the final marginal cost of $\underline{u}_{3}$ is zero; this vector can be brought into the basis yielding a new best approximation which interpolates the first and fifth data points. Other best approximations can be generated as convex combinations of these two extreme point solutions.

This concludes the description of our primal algorithm. There are, of course, several variants of the standard form of the simplex method which can be used to solve a linear programming problem (two forms of the revised simplex method, the primal-daul algorithm, the dual simplex algorithm, etc.). However, the denseness of the condensed tableau corresponding to (2), the availability of an initial basic feasible solution, and the simplicity with which we can implement our main modification, combine together to make the standard form of the simplex method the most economical algorithm for the $\&_{1}$ problem.

\section{Numerical results}

The following test problems were designed both to illustrate the flexibility of the various algorithms and to compare their computational behavior.

Example 1. Approximate $f(x)=e^{-x} \sin x$ on $x=\{0(0.02) 4\}$ by $L(A, x)=\sum_{j=1}^{n} a_{j} x^{j-1}$ for $n=1,2, \ldots, 7$.

Example 2. Approximate $f(x, y)=\operatorname{Re}\left\{\int_{x+i y}^{\infty} e^{-z / z d z}\right\}$ on 100 points defined by $x=0.1(0.1) 1.0, y=0.1(0.1) 1.0$, using the two-dimensional approximating function

$$
L(A, x, y)=a_{1}+a_{2} x+a_{3} y+a_{4} x y+a_{5} x^{2}+a_{6} y^{2}
$$


(The values of $f(x, y)$ are taken from Fox, Goldstein and Lastman [8] where they are recorded to six decimal places).

Example 3. Approximate $f(x)=\sqrt{x}$ on $X=\{0(0.02) 1\}$ by the cubic spline $L(A, x)=\sum_{j=1}^{4} a_{j} x^{j-1}+\sum_{t=1}^{4} a_{4+t}\left(x-\alpha_{t}\right)^{3}$, where $\alpha_{1}=0.1, \alpha_{2}=0.2, \alpha_{3}=0.4$,
$\alpha_{4}=0.7$.

Example 4. Approximate $f(x)=\sin x$ on $X=\left\{-\frac{\pi}{2}\left(\frac{\pi}{k}\right) \frac{\pi}{2}\right\}$, for $k=20$, $50,100,200$, by $L(A, x)=P_{5}(x)=\sum_{j=1}^{6} a_{j} x^{j-1}$ with $P_{5}^{\prime}\left(-\frac{\pi}{2}\right)=0, P_{5}^{\prime}\left(\frac{\pi}{2}\right)=0$. The constraints on the derivative can be handled by regarding $\left(-\frac{\pi}{2}, 0\right)$ and $\left(\frac{\pi}{2}, 0\right)$ as two data points to be approximated by $P_{5}^{\prime}(x)$. We then ensure that the derivative actually interpolates these points by suitably weighting the two extra rows corresponding to these constraints. (We simply multiplied both rows by 1000 in the initial tableau).

Example 5. Approximate $f(x)=\min \left(e^{x}, e^{1 / 2}\right)$ on $x=\left\{0\left(\frac{1}{d}\right) 1\right\}$, for $d=20,50,100,200$, by $L(A, x)=a_{1}+\sum_{j=1}^{5}\left(a_{2 j} \sin j x+a_{2 j+1} \cos j x\right)$.

Example 6. Calculate a best $\ell_{1}$ solution to the following overdetermined system of linear equations: 


$$
\begin{aligned}
& 5 a_{1}+3 a_{2}+4 a_{3}+12 a_{4}+4 a_{5}=7 \\
& 9 a_{1}+7 a_{2}+3 a_{3}+19 a_{4}+13 a_{5}=4 \\
& 6 a_{1}+6 a_{2}+0 a_{3}+12 a_{4}+12 a_{5}=2 \\
& 9 a_{1}+9 a_{2}+7 a_{3}+25 a_{4}+11 a_{5}=7 \\
& 3 a_{1}+0 a_{2}+1 a_{3}+4 a_{4}+2 a_{5}=7 \\
& 8 a_{1}+1 a_{2}+8 a_{3}+17 a_{4}+1 a_{5}=7 \\
& 1 a_{1}+9 a_{2}+8 a_{3}+18 a_{4}+2 a_{5}=3 \\
& 0 a_{1}+9 a_{2}+3 a_{3}+12 a_{4}+6 a_{5}=3 \\
& 3 a_{1}+1 a_{2}+1 a_{3}+5 a_{4}+3 a_{5}=5 \\
& 6 a_{1}+7 a_{2}+6 a_{3}+19 a_{4}+7 a_{3}=1 \\
& 6 a_{1}+1 a_{2}+9 a_{3}+16 a_{4}+-2 a_{5}=4 \\
& 0 a_{1}+4 a_{2}+8 a_{3}+12 a_{4}+-4 a_{5}=1 \\
& 0 a_{1}+5 a_{2}+7 a_{3}+12 a_{4}+-2 a_{5}=6 \\
& 7 a_{1}+3 a_{2}+2 a_{3}+12 a_{4}+8 a_{5}=6 \\
& 5 a_{1}+4 a_{2}+9 a_{3}+18 a_{4}+0 a_{5}=0
\end{aligned}
$$

The entries in the first, second, and third columns were chosen from a table of random integers. The fourth column is the sum of the previous three columns, and the fifth column is the sum of the first two columns minus the third column. The matrix of coefficients $\Phi$ is thus a $15 \times 5$ matrix of column rank 3 . The 
entries on the right-hand side, which correspond to values of $f(x)$ in the approximation problem, were also chosen from a table of random integers.

Example 7. Approximate $f(x)=1+x+x^{2}+x^{3}+x^{4}+E(x)$, where $E(x)=5$ for $0.94 \leq x \leq 1$ and $E(x)=0$ otherwise, on $X=\{0(0.02) 1\}$ by $L(A, x)=\sum_{j=1}^{5} a_{j} x^{j-1}$. This example was designed primarily to test the effectiveness of the new algorithm of Usow [14]. If an intermediate approximation is formed from just the uncontaminated portion of the data, corresponding to the range $0 \leq x \leq 0.92$, it will result in a large number of zero residuals (i.e. an $L(A, x)$ is formed which interpolates $f(x)$ in many more than $n \quad(=5)$ points of X). This phenomenon can cause the original algorithm of Usow [13] to stop prematurely. In a primal linear programming algorithm it causes degeneracy; in practice the simplex method handles degeneracy without any special provisions having to be made.

We attempted to solve all seven test examples using each of the four methods identified as:

(i) USOW: see Usow [14].

(ii) ROB2: see Robers and Robers [12].

(iii) DUAL: the dual formulation (3) solved by the bounded-variable simplex method of Dantzig [7].

(iv) PRIM: the primal formulation (2) solved by our algorithm Methods (i) and (ii) were coded by their respective authors, and methods (iii) and (iv) were coded by us; all of the programs were written in Fortran IV. The 
program supplied by Dr. K. H. Usow is still es sentially at the experimental stage, since there are some quite delicate computations involved in his method. Unfortunately, we were not able to solve some of our test problems using his program even though all seven problems were attempted.

The central processor times given in Table 4 are for a CDC 6600 computer, and they do not include time spent in compiling or setting up the data for each problem. (These CP times are not exact, for we have observed slight discrepancies when repeating some of the computations). The calculations were performed in single precision floating-point arithmetic using a 48-bit mantissa. The best $\ell_{1}$ error of approximation given in the final column of Table 4 is from the output supplied by our algorithm PRIM. The algorithm DUAL produced essentially the same results as PRIM, but ROB2 sometimes differed from these two by somewhat more than the expected rounding error (particularly in Example 5). The error of approximation produced by Usow also differed in some cases from that shown in Table 4. In the linear programming algorithms we used a tolerance of $10^{-8}$ when deciding whether or not certain quantities are "zero", and the initial approximation required by Usow was chosen by interpolating the first $n$ points of $X$.

Finally, we note that all of these test problems have unique best $\ell_{1}$ approximations (except, of course, for the linearly dependent overdetermined system in Example 6). 


\begin{tabular}{|c|c|c|c|c|c|}
\hline \multirow{2}{*}{ Example } & \multicolumn{4}{|c|}{ TIME (seconds) } & \multirow{2}{*}{$\begin{array}{l}\text { Error of Approximation } \\
\sum_{i=1}^{m}\left|f\left(x_{1}\right)-L\left(A^{*}, x_{i}\right)\right|\end{array}$} \\
\hline & USOW & $\mathrm{ROB} 2$ & DUAL & PRIM & \\
\hline $\begin{array}{c}1(m=201) \\
n=1\end{array}$ & * & 2.38 & 0.63 & 0.29 & 21.81556 \\
\hline $\mathrm{n}=2$ & 2.04 & 13.95 & 2.79 & 0.85 & 9.27171 \\
\hline $\mathrm{n}=3$ & 1.97 & 18.57 & 2.58 & 0.81 & 9.07895 \\
\hline $\mathrm{n}=4$ & 2.61 & 30.00 & 4.21 & 1.69 & 4.64073 \\
\hline$n=5$ & 2.81 & 40.82 & 5.12 & 2.54 & 1.05559 \\
\hline$n=6$ & 7.46 & 54.76 & 6.09 & 3.55 & 0.06045 \\
\hline$n=7$ & 5.94 & 61.77 & 7.63 & 3.29 & 0.03471 \\
\hline $2(m=100, n=6)$ & $*$ & 16.93 & 1.68 & 1.06 & 3.87741 \\
\hline $\begin{array}{l}3(m=51, n=8) \\
4(n=6)\end{array}$ & \multicolumn{5}{|c|}{$4(n=6)$} \\
\hline$m=23$ & 0.18 & 0.39 & 0.12 & 0.05 & 0.00188 \\
\hline$m=53$ & 0.65 & 1.39 & 0.60 & 0.17 & 0.00436 \\
\hline$m=103$ & 1.57 & 6.28 & 2.10 & 0.51 & 0.00858 \\
\hline$m=203$ & 5.25 & 33.18 & 7.86 & 1.67 & 0.01694 \\
\hline \multicolumn{6}{|l|}{$5(n=11)$} \\
\hline$m=21$ & 0.85 & $*$ & 0.28 & 0.15 & 0.06170 \\
\hline$m=51$ & 1.60 & * & 1.60 & 0.51 & 0.15642 \\
\hline$m=101$ & 4.42 & $*$ & 4.24 & 1.33 & 0.30852 \\
\hline$m=201$ & 10.90 & $*$ & 15.54 & 4.27 & 0.61230 \\
\hline $6(m=15, n=5)$ & $*$ & * * & 0.05 & 0.02 & 34.22449 \\
\hline $7(m=51, n=5)$ & $*$ & 2.80 & 0.28 & 0.22 & 16.73658 \\
\hline
\end{tabular}

* No results available

TABLE 4: A comparison of the calculation times required by each algorithm to solve the test problems on a CDC 6600 computer. 


\section{Remarks}

It is quite clear that our new primal algorithm is both efficient and reliable, and we supply a Fortran IV program for the method in the Appendix. We have used a double precision version of this code to solve several other problems on an IBM $360 / 50$ and on a Univac 1108 computer. (In particular, we have solved an overdetermined system of size $500 \times 10$ using double precision versions of PRIM and DUAL on a Univac 1108; PRIM took 4.56 seconds and DUAL required 59.68 seconds to solve this problem).

In practice, of course, it makes little difference whether (say) PRIM or DUAL is used to solve a single small problem in data analysis. However, it is important to have an efficient algorithm at hand if several tens or hundreds of problems are to be solved. An important instance of this occurs when nonlinear approximating functions are used to interpret data, for in this case some linearization of the problem will normally be employed which calls for the repeated application of a linear algorithm. Two recent methods along this line are described in Barrodale, Roberts and Hunt [5] and Osborne and Watson [9].

\section{Acknowledgements}

We are very grateful to Dr. Philip Robers and to Dr. Karl Usow for supplying us with programs for their algorithms. Mr. Fred Crary and Mr. Bruce Wilson provided us with excellent programming assistance. The financial support provided by NRC Grants A5251 and A7143 is also much appreciated. 


\section{REFERENCES}

1. N. N. Abdelmalek: Linear $L_{1}$ Approximation for a Discrete Point Set and $L_{1}$ Solutions of Overdetermined Linear Equations. J. ACM, 18 (1971), $41-47$.

2. I. Barrodale: $L_{1}$ Approximation and the Analysis of Data. Applied Stat., $17(1968), 51-57$.

3. I. Barrodale: On Computing Best $\mathrm{L}_{1}$ Approximations. From Approximation Theory, A. Talbot (ed.), Academic Press (1970), 205-215.

4. I. Barrodale and F. D.K. Roberts: Applications of Mathematical Programming to $\ell_{\mathrm{p}}$ Approximation. From Nonlinear Programming, J. B. Rosen, O..L. Mangasarian and K. Ritter (eds.), Academic Press $(1970), 447-464$.

5. I. Barrodale, F. D. K. Roberts and C. R. Hunt: Computing Best $\ell_{p}$ Approximations by Functions Nonlinear in One Parameter. Comp. J., $13(1970), 382-386$.

6. I. Barrodale and A. Young: Algorithms for Best $\mathrm{L}_{1}$ and $\mathrm{L}_{\infty}$ Linear Approximations on a Discrete Set. Numer. Math., $\underline{8}$ (1966), 295-306.

7. G. B. Dantzig: Linear Programming and Extensions. Princeton Univ. Press (1963).

8. P. Fox, A. A. Goldstein and G. Lastman: Rational Approximations on Finite Point Sets. From Approximation of Functions, H. L. Garabedian (ed.), Elsevier (1965), 57-67. 
9. M. R. Osborne and G. A. Watson: On an Algorithm for Discrete Nonlinear $\mathrm{L}_{1}$ Approximation. Comp. J., 14 (1971), 184-188.

10. P. Rabinowitz: Applications of Linear Programming to Numerical Analysis. SIAM Rev., 10 (1968), 121-159.

11. P. D. Robers and A. Ben-Israel: An Interval Programming Algorithm for Discrete Linear $L_{1}$ Approximation Problems. J. Approx. Th., 2 (1969), $323-336$

12. P. D. Robers and S. S. Robers: Discrete Linear $L_{1}$ Approximation by Interval Linear Programming. (To appear in an ACM publication).

13. K. H. Usow: On $\mathrm{L}_{1}$ Approximation II; Computation for Discrete Functions and Discretization Effects. SIAM J. Numer. Anal., 4 (1967), 233-244.

14. K. H. Usow: An Algorithm for Discrete Linear $L_{1}$ Approximation with Application to Spline Functions. (Manuscript).

15. H. M. Wagner: Linear Programming Techniques for Regression Analysis. J. Amer. Statist. Assoc., 54 (1959), 206-212. 


\section{APPENDIX}

The Fortran IV SUBROUTINE $\mathrm{L}_{1}$ (Q, M3, N2, TOLER, SCALE)

This subroutine calculates (for $\mathrm{n} \leq \mathrm{m}$ ) a best $\ell_{1}$ approximation to $\mathrm{f}(\mathrm{x})$ by $\sum_{j=1}^{n} a_{j} \phi_{j}(x)$ on a set $x=\left\{x_{1}, x_{2}, \ldots, x_{m}\right\}, i_{.} e$. parameters $a_{1}^{*}, a_{2}^{*}, \ldots, a_{n}^{*}$ are determined which minimize $\sum_{i=1}^{m}\left|f\left(x_{i}\right)-\sum_{j=1}^{n} a_{j} \phi_{j}\left(x_{i}\right)\right|$. The algorithm is a modification of the standard form of the simplex method applied to the primal formulation of the $\ell_{1}$ problem as a linear program.

INPUT

Q: A real array of dimensions $(m+3, n+2)$ which initially contains the $m \times n$ matrix

$$
\Phi=\left(\begin{array}{cccc}
\phi_{1}\left(\mathrm{x}_{1}\right) & \phi_{2}\left(\mathrm{x}_{1}\right) & \cdots & \phi_{\mathrm{n}}\left(\mathrm{x}_{1}\right) \\
\phi_{1}\left(\mathrm{x}_{2}\right) & \phi_{2}\left(\mathrm{x}_{2}\right) & \cdots & \phi_{\mathrm{n}}\left(\mathrm{x}_{2}\right) \\
\vdots & \vdots & & \vdots \\
\phi_{1}\left(\mathrm{x}_{\mathrm{m}}\right) & \phi_{2}\left(\mathrm{x}_{\mathrm{m}}\right) & \cdots & \phi_{\mathrm{n}}\left(\mathrm{x}_{\mathrm{m}}\right)
\end{array}\right)
$$

in the first $m$ rows and $n$ columns, and the vector of function values $\left[f\left(x_{1}\right), f\left(x_{2}\right), \ldots, f\left(x_{m}\right)\right]^{T}$ in the first $m$ rows of the $(n+1)$ st. column. No other input is required.

M3: A positive integer set equal to $m+3$.

N2: A positive integer set equal to $n+2$. 
TOLER: A positive real number set to approximately the desired order of relative accuracy of the calculated parameters $a_{j}^{*}, e . g .10^{-5}$ or $10^{-8}$. This number prevents pivoting on elements of $Q$ which are too small, and a decrease in its value will usually cause more iterations of the simplex method.

SCALE: An integer that should be set to 1 only if the user wishes the initial data to be scaled columnwise. In this event, the final tableau is rescaled automatically. (Scaling is usually beneficial).

No other input to the subroutine is required. If a best weighted $\ell_{1}$ approximation is desired, the user should multiply each row of data by its chosen (positive) weight prior to entering the subroutine.

\section{OUTPUT}

All output parameters are real numbers contained in $Q$. $Q(m+2, n+1): \quad$ Has the value 1 if a best approximation has been calculated, and the value 2 if no suitable pivot is available and the iterations have been terminated prematurely (this latter condition can occur only with badly conditioned problems).

$Q(m+1, n+1): \quad$ Error of approximation $\sum_{i=1}^{m}\left|f\left(x_{i}\right)-\sum_{j=1}^{n} a_{j}^{*} \phi_{j}\left(x_{i}\right)\right|$.

$Q(m+1, n+2): \quad$ The rank of the matrix $\Phi$. (If the rank of $\Phi$ is less than $n$, the surplus columns of $\Phi$ are stored in the first columns of $Q$ ). $Q(m+2, n+2): \quad$ The number of iterations required by the simplex method. $\mathrm{Q}(\mathrm{m}+3, \mathrm{n}+2): \quad$ Has the value $l$ if the best approximation is unique and the value 0 if there is more than one best approximation (which may be due to the rank of $\Phi$ being less than $n$ ). 
The absolute values of the parameters $a_{1}^{*}, a_{2}^{*}, \ldots, a_{n}^{*}$ are contalned in the first $n$ rows of the $(n+1)$ st. column, and their signed labels are contained in the corresponding rows of the $(n+2)$ nd. column $\left(a_{1}^{*}\right.$ has the label $\pm 1, a_{2}^{*}$ has the label \pm 2 , etc.). The absolute values of $(m-n)$ of the deviations $f\left(x_{i}\right)-\sum_{j=1}^{n} a_{j}^{*} \phi_{j}\left(x_{i}\right)=u_{i}-v_{i}=d_{i}$ are contained in the remaining $(m-n)$ rows of the $(n+1)$ st. column, and their signed labels are contained in the corresponding rows of the $(n+2)$ nd. column $\left(d_{1}\right.$ has the label $\pm\left(10^{6}+1\right), d_{2}$ has the label $\pm\left(10^{6}+2\right)$, etc. $)$. Thus, for the worked example given in Table 3 of this paper, the first $m$ rows of the $(n+1) s t$. and $(n+2) n d$. columns of $Q$ finally appear as:

\begin{tabular}{c|c}
$1 / 2$ & 1 \\
$1 / 2$ & 2 \\
$1 / 2$ & $-\left(10^{6}+2\right)$ \\
$1 / 2$ & $10^{6}+4$ \\
1 & $-\left(10^{6}+5\right)$
\end{tabular}

If the labels $\pm\left(10^{6}+i\right)$ do not appear in the $(n+2)$ nd. column, then $d_{i}=0$ and $x_{1}$ is the abscissa of a point of interpolation. 
00000000000000000000000000000000000

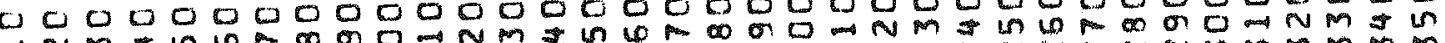

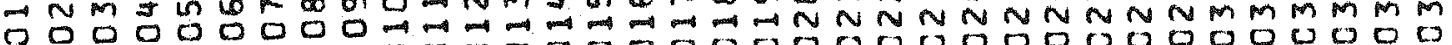

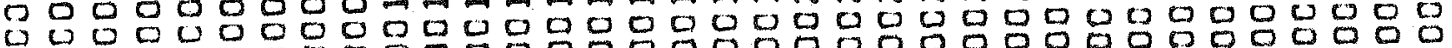
$0008000000000000000000000000000 \%$

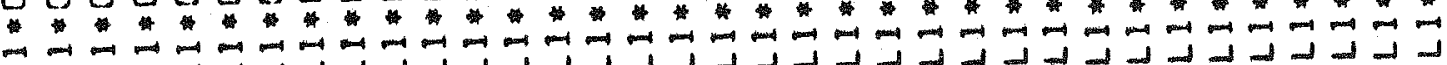

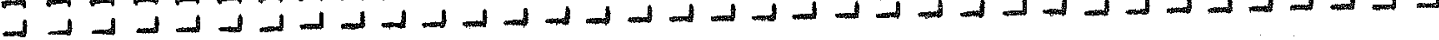

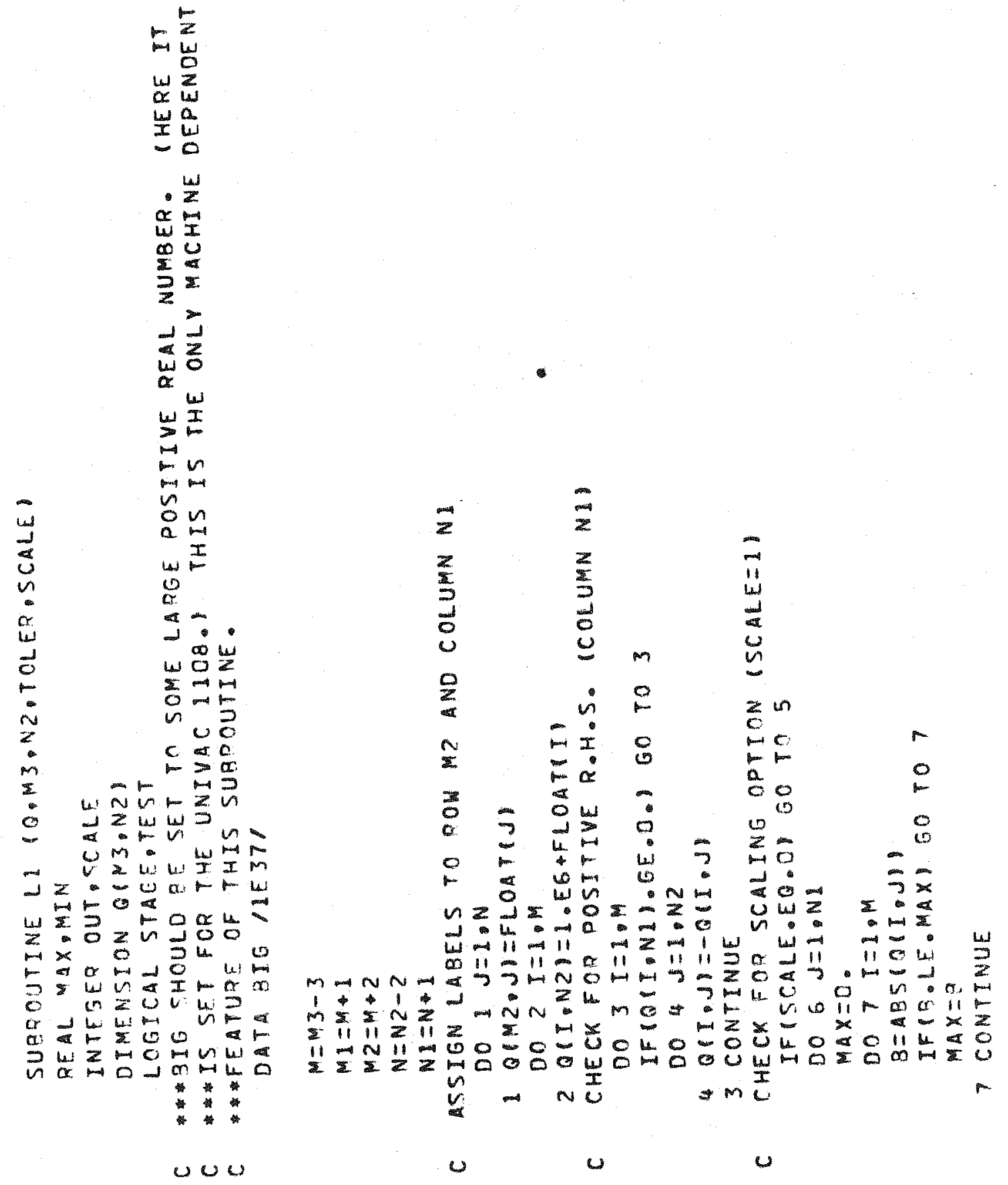


00000000000000000000000000000000000 W

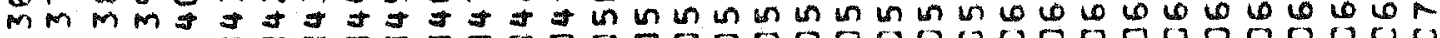

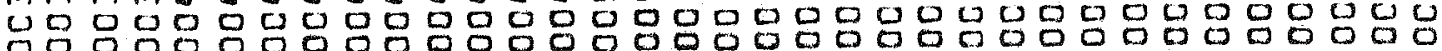

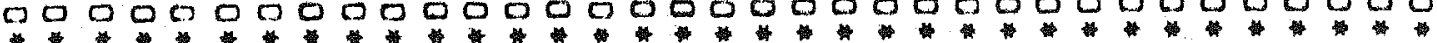
แ 던

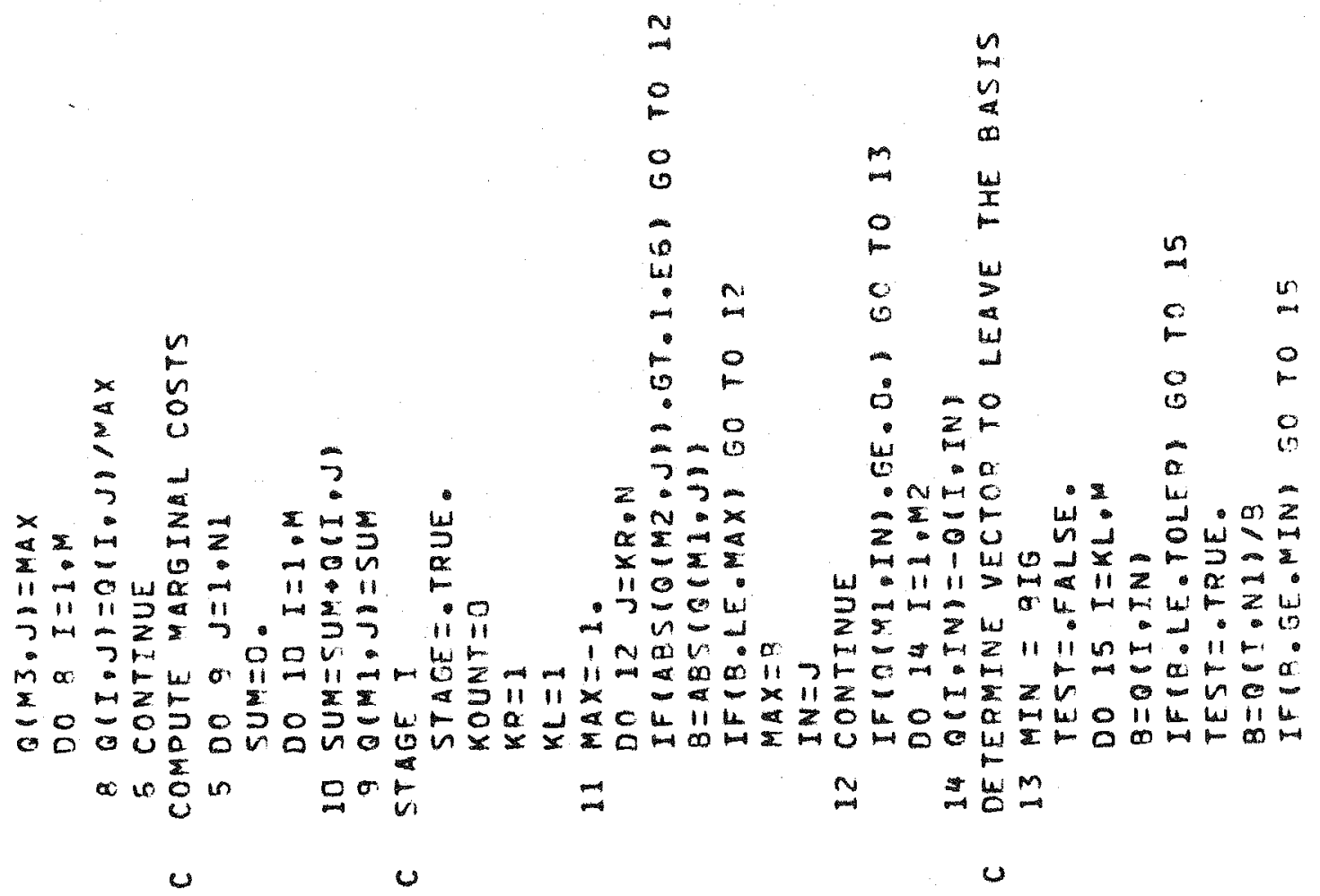


00000000000000000000000000000000000 TNMJULA

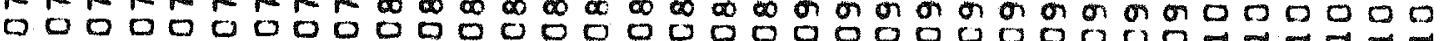

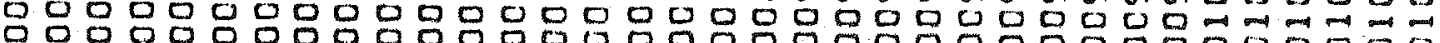
* o 0000000000000000000000000000000

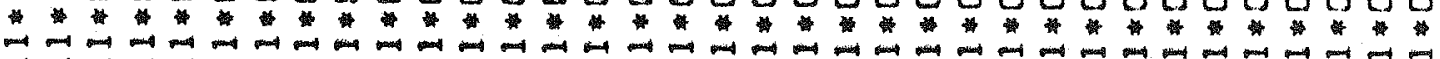
Jد

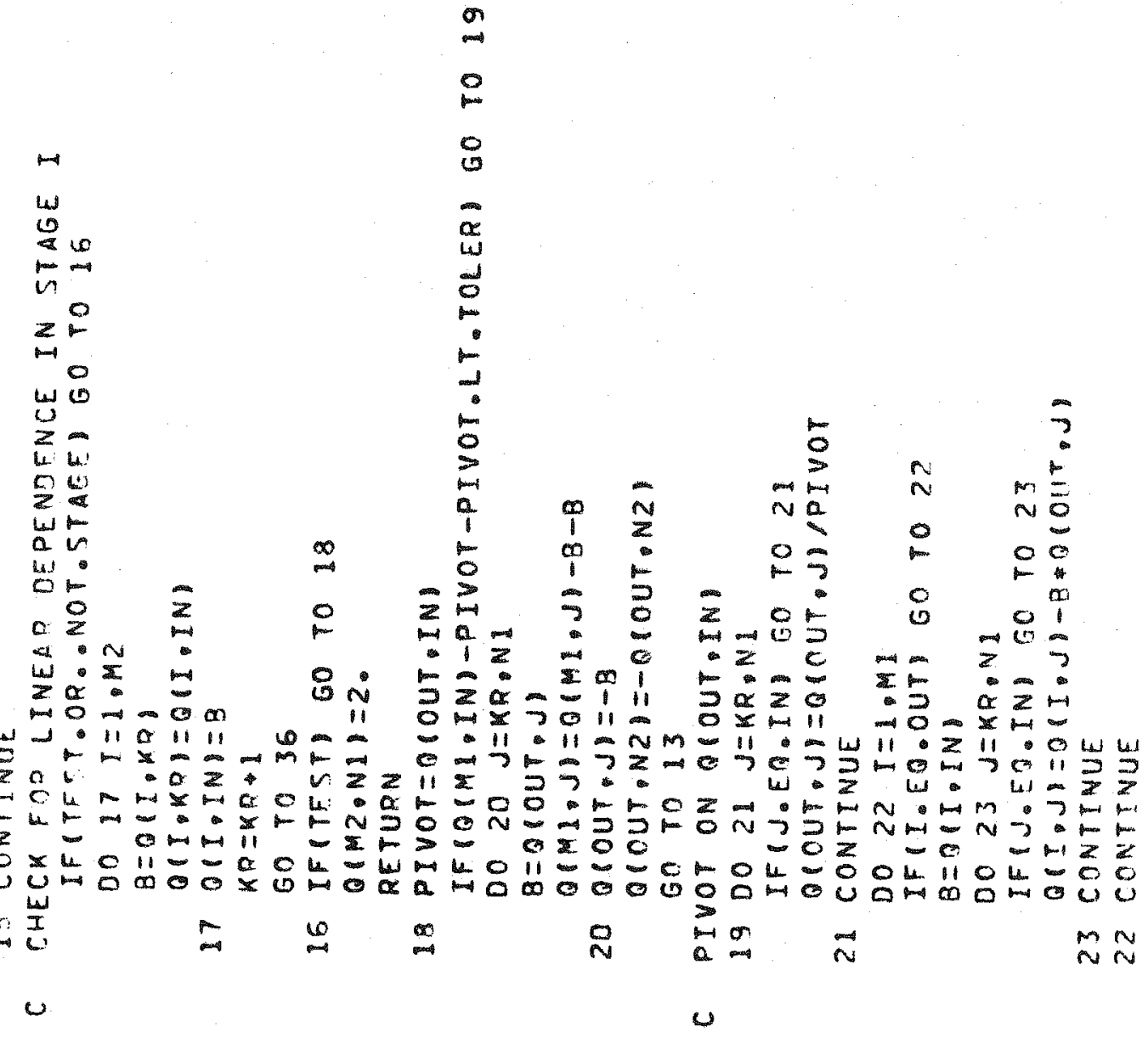


00000000000000000000000000000000000

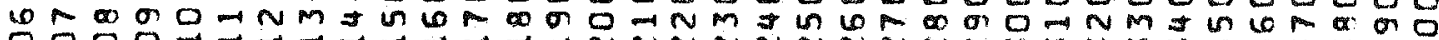

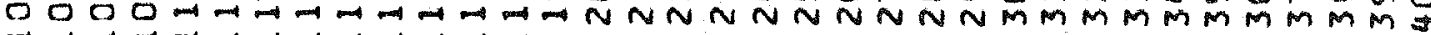

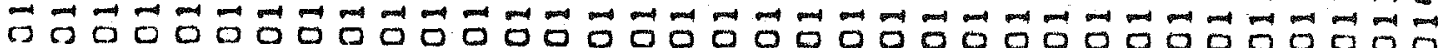
* * * * * * * * a

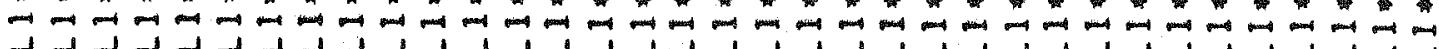

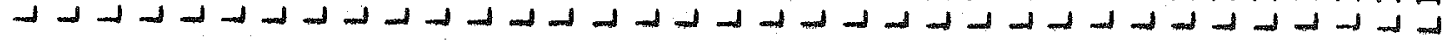

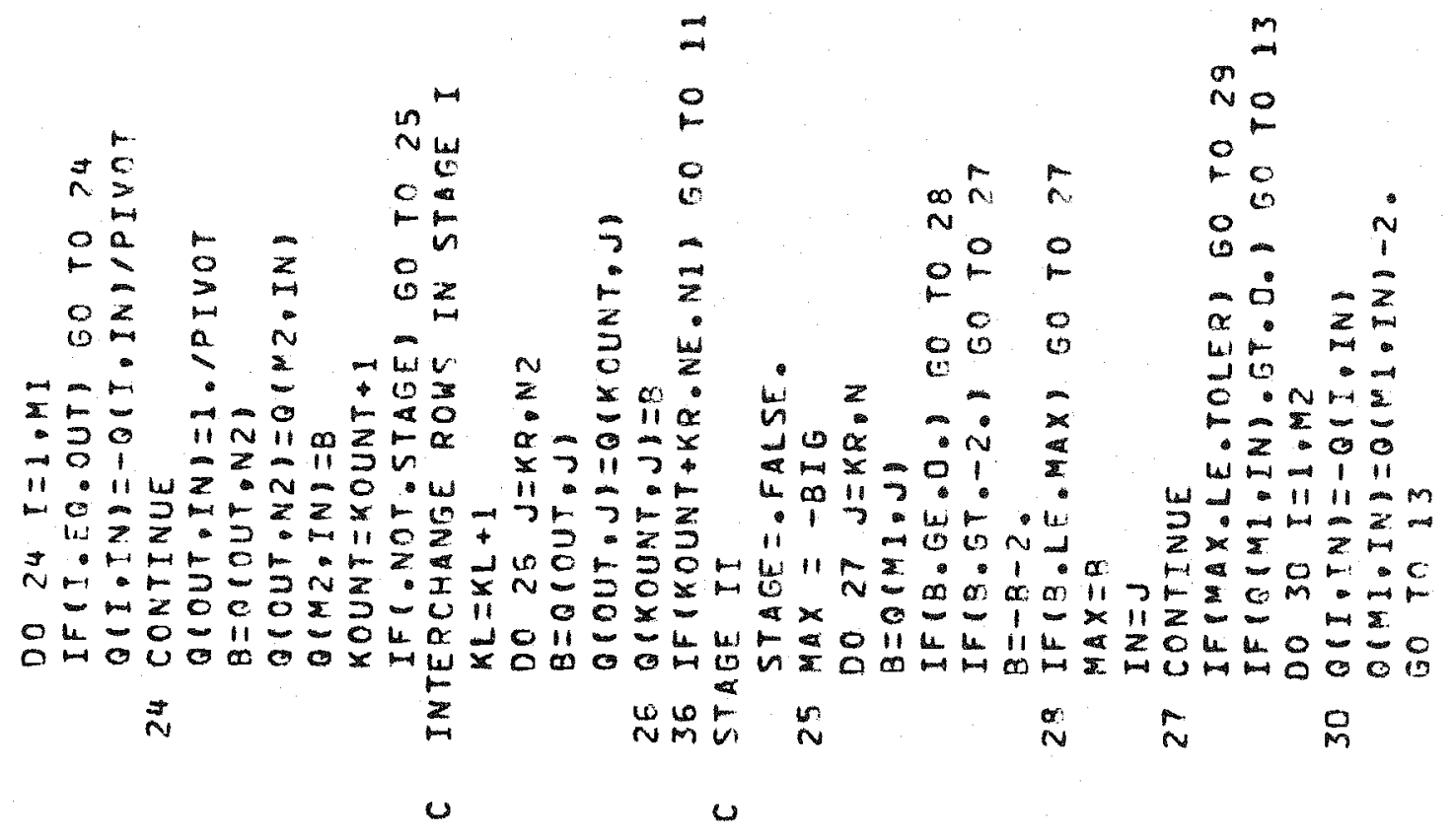


0000000000000000000000000000

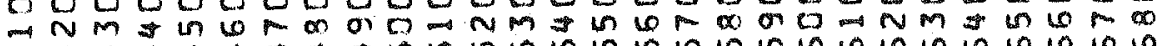

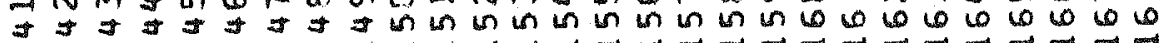

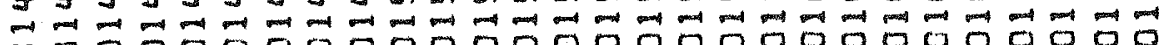
6000000000000000000000000000 - \#

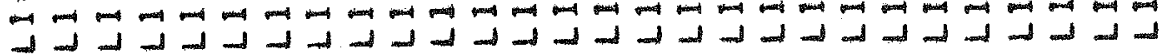

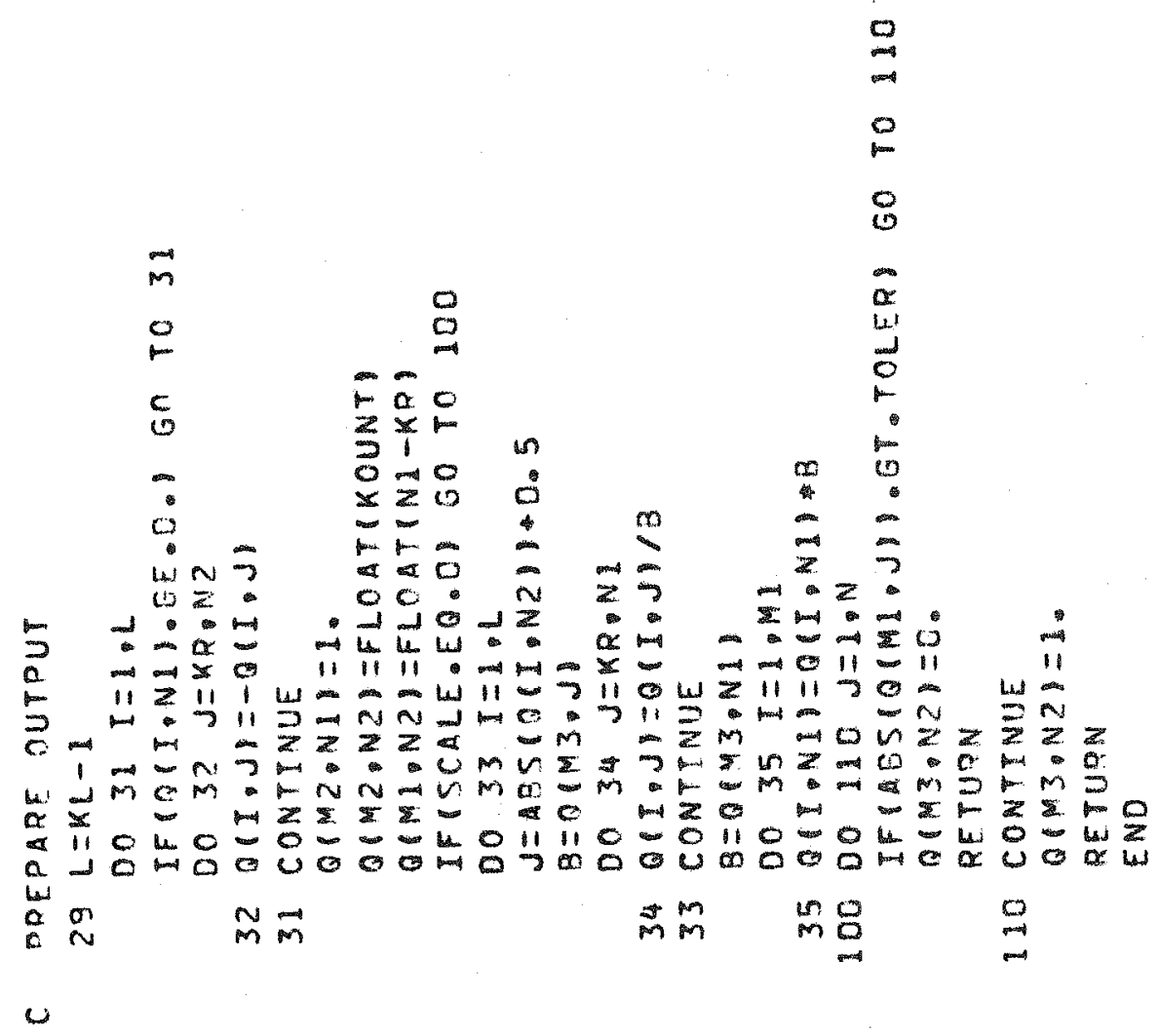

